\title{
Yield and Quality of White Jute (Var. Bjri Deshi Pat 8) Seed as Influenced by LinexPlant Spacing in Different Growing Areas of Bangladesh
}

\author{
*Md. Mahbubul Islam, Md. Shahadat Hossain, Md. Saheb Ali \\ Md. Siddiqur Rahman
}

Agronomy Division, Bangladesh Jute Research Institute, Manik Mia Ave., Dhaka-1207

${ }^{*}$ Chief Scientific Officer, Agronomy Division, Bangladesh Jute Research Institute, Manik Mia Ave., Dhaka-1207 mahbub_agronomy@yahoo.com

Abstract: The experiment was conducted at JAES, Manikganj, JRRS, Rangpur and JRSS, Jessore during 2015-16 to determine the optimum plant spacing of BJRI Deshi Pat-8 (BJC-2197) for seed production at late season. The experiment was laid out in RCBD design with three replications. Different plant spacing viz., $30 \mathrm{~cm} \times 10 \mathrm{~cm}$, $30 \mathrm{~cm} \times 12.5 \mathrm{~cm}, 30 \mathrm{~cm} \times 15 \mathrm{~cm}, 35 \mathrm{~cm} \times 10 \mathrm{~cm}, 35 \mathrm{~cm} \times 12.5 \mathrm{~cm}, 35 \mathrm{~cm} \times 15 \mathrm{~cm}, 40 \mathrm{~cm} \times 10 \mathrm{~cm}, 40 \mathrm{~cm} \times 12.5 \mathrm{~cm}$, $40 \mathrm{~cm} \times 15 \mathrm{~cm}, 45 \mathrm{~cm} \times 10 \mathrm{~cm}, 45 \mathrm{~cm} \times 12.5 \mathrm{~cm}$ and $45 \mathrm{~cm} \times 15 \mathrm{~cm}$ were used as treatment. The crop was harvested at proper maturity of the pods of plant as per BJRI recommendation. Results revealed that all the yield and quality contributing characters were differed significantly except branch plant ${ }^{-1}, 1000$ seeds weight and Moisture percentage due to different plant spacing. The highest seed yields and germination $1398.33 \mathrm{kgha}^{-1}$ and $92.33 \%$ at Manikganj, $1164.73 \mathrm{kgha}^{-1}$ and $92.67 \%$ at Rangpur and; $1197.00 \mathrm{kgha}^{-1}$ and $91.33 \%$ at Jessore, respectively were obtained from the spacing treatment $35 \mathrm{~cm} \times 12.5 \mathrm{~cm}$.

Keywords: White jute, seed, yield, quality, spacing.

Abbreviations: JAES, Jute Agriculture Experimental Station; JRRS, Jute Regional Research Station.

\section{INTRODUCTION}

Jute is a cash crop grown in the summer season (Kharif ${ }^{-1}$ ) of Bangladesh. In 2010-11, 0.803 million ha land cultivated for jute. Production of raw jute was 1.5 million MT by volume in 2010-11. Jute cultivation area was $6 \%$ of total land area of 13 million ha, of which 8.44 million ha belongs to agricultural land. Jute cultivation area was $10 \%$ of agricultural land area. Jute production was $26 \%$ as of all agricultural crops. Annual jute seed requirement was 5,000-5,500 MT. Jute seed supply were from i) Public: 2,187 MT and from ii) Import 3,617 MT in 2010-2011(IJSG, 2012).

In northern districts like Dinajpur, Rangpur 15 days earlier than that of southern (Jessore, kustia) (Islam 2009). The varieties 0-4, 0-9897, and chaitali sown on 1 and 15 August produced as much as 990 and 935 kg seeds per hectare (Khan, 1997). In C. olitorius L. jute variety OM-1, Seed yield plant ${ }^{-1}, 1000$ - seed wt. and germinatio.n differed significantly due to sowing dates (Chanda, 1999). Jute seed crop could be sown form may 15 to September 30 (Talukder, 2001). Planting time (July 15 to September 15) of seeds of 0-72 olitorius jute differed significantly for seed yield (Alom, 2010). The optimum time of late jute seed production was June-July for $C$. capsularis L. white August-September for C. olitorius L. (Islam, 2010). To get optimum plant population and desired yield, seeding rate was optimized and found that seeds having $80 \%$ viability, $5 \mathrm{kgha}^{-1}$ of tossa jute, $7 \mathrm{kgha}^{-1}$ of deshi and 12-15 kgha-1 of Kenaf and Mesta seeds could offer desired population and optimum yield. For seed crop seed rate depends on the soil condition and planting methods followed. As a thumb rule, for Deshi jute $4.0-4.5 \mathrm{~kg} / \mathrm{ha}$ in line sowing and $5.0-5.5 \mathrm{~kg} / \mathrm{ha}$ in broadcast sowing should to be maintained. On the other 
Yield and Quality of White Jute (var. BJRI Deshi Pat 8) Seed as Influenced by LinexPlant Spacing in Different Growing Areas of Bangladesh

hand for Tossa jute 3.0-3.5 kg/ha in line sowing and 4.0-4.5 kg/ha in broadcast sowing should be maintained. As jute seeds have no other alternative uses, it is to sow even if it is of $50 \%$ viability by adjustment the seed rate (Islam and Rahman, 2008).

The conventional practice of jute seed production is to keep a part of the fibre crop for seed harvest. Mostly we cannot maintain the line to line as well as plant to plant spacing. In case of seed production of jute, spacious environment is very much important for production of maximum number of branches and pods, resulting higher amount of quality seed, where jute plants get enough space to develop canopy horizontally. Jute plants able to grow many branches. However, maintaining the spacing in seed production of jute crop at late season is very much important. In order to improve the yield and quality of seed the experiment is designed to assess the effect of different spacing on seed production of white jute (Corchorus capsularis L.) in late season.

\section{Materials ANd Methods}

The experiment was conducted at Jute Agriculture Experimental Station (JAES), Manikganj, Jute Research Regional Station (JRRS), Rangpur and Jute Research Sub Station (JRSS), Jessore during 2015-16 to determine the optimum plant spacing of newly released variety BJRI Deshi Pat 8 (line: BJC-2197). Different plant spacing viz., $30 \mathrm{~cm} \times 10 \mathrm{~cm}$ ("row to row spacing $30 \mathrm{~cm}$ " and "plant to plant spacing $10 \mathrm{~cm}$ "), $30 \mathrm{~cm} \times 12.5 \mathrm{~cm}$, $30 \mathrm{~cm} \times 15 \mathrm{~cm}, 35 \mathrm{~cm} \times 10 \mathrm{~cm}, 35 \mathrm{~cm} \times 12.5 \mathrm{~cm}, 35 \mathrm{~cm} \times 15 \mathrm{~cm}, 40 \mathrm{~cm} \times 10 \mathrm{~cm}, 40 \mathrm{~cm} \times 12.5 \mathrm{~cm}, 40 \mathrm{~cm} \times 15 \mathrm{~cm}, 45 \mathrm{~cm} \times 10 \mathrm{~cm}$, $45 \mathrm{~cm} \times 12.5 \mathrm{~cm}$ and $45 \mathrm{~cm} \times 15 \mathrm{~cm}$ were used as treatment. The experiment was laid out in RCBD design with three replications. Unit plot size would be $4.0 \mathrm{~m} \times 2.5 \mathrm{~m}$. Space between plot to plot and around the field $1.0 \mathrm{~m}$ and between replications $1.5 \mathrm{~m}$ was maintained. Seeds were sown in assigned spacing according to treatment at late season (last week of August to $1^{\text {st }}$ week of September) (Islam, 2010). Other cultural and intercultural practices were attended as per BJRI recommendation. Location wise average data of seed yield and quality attributing characters were analyzed with the help of computer statistical package (MSTAT). The mean differences among the treatments were adjusted as per Duncan's Multiple Range Test (DMRT) and T-test at 0.05 level (Gomez and Gomez, 1984).

\section{RESULTS AND Discussion}

Results revealed that all the yield and quality contributing characters were differed significantly except Branch plant $^{-1}, 1000 \mathrm{SW}(\mathrm{g})$ and Moisture percentage due to different plant spacing (Table 1). The highest yield $1398.33 \mathrm{kgha}^{-1}$ was obtained at Manikganj from the spacing $35 \mathrm{~cm} \times 12.5 \mathrm{~cm}$ which was followed by the spacing $40 \mathrm{~cm} \times 10.05 \mathrm{~cm}\left(1379.33 \mathrm{kgha}^{-1}\right)$ and $30 \mathrm{~cm} \times 15 \mathrm{~cm}\left(1341.00 \mathrm{kgha}^{-1}\right)$. All the yield and yield contributing characters differed significantly except branch plant ${ }^{-1}$ due to different spacing. Yield contributing characters were gave similar results according to the seed yield at Manikganj (Table 1). Germination percent differed significantly due to spacing. However, $1000 \mathrm{SW}$ (g) and moisture percentage were insignificant due to different spacing. The highest germination was observed $92.33 \%$ at Manikganj from $35 \mathrm{~cm} \times 12.5 \mathrm{~cm}$ specing which was followed by the spacing $40 \mathrm{~cm} \times 10.0 \mathrm{~cm}(91.67 \%)$ and $30 \mathrm{~cm} \times 15 \mathrm{~cm}(91.33 \%)$ (Table 2). The results were in agreement with Islam (2010) and Islam (2009).

Table1. Effect of spacing on seed yield and yield contributing characters of white jute at Manikganj

\begin{tabular}{|c|c|c|c|c|c|c|}
\hline \multicolumn{2}{|c|}{ Treatment (Spacing) } & \multirow{2}{*}{$\begin{array}{c}\text { Branch } \\
\text { plant }\end{array}$} & \multirow{2}{*}{$\begin{array}{c}\text { Pods } \\
\text { plant }^{-1}\end{array}$} & $\begin{array}{c}\text { Seed } \\
\text { pod }^{-1}\end{array}$ & $\begin{array}{c}\text { SW } \\
\text { plant }^{-1} \text { (g) }\end{array}$ & SY (kgha-1 $)$ \\
\hline$S_{1}$ & $30 \mathrm{~cm} \times 10.0 \mathrm{~cm}$ & 4.08 & 38.96 & 27.56 & 5.36 & 1141.00 \\
\hline$S_{2}$ & $30 \mathrm{~cm} \times 12.5 \mathrm{~cm}$ & 5.74 & 56.63 & 33.88 & 7.16 & 1302.67 \\
\hline$S_{3}$ & $30 \mathrm{~cm} \times 15.0 \mathrm{~cm}$ & 5.34 & 70.43 & 35.42 & 8.89 & 1341.00 \\
\hline$S_{4}$ & $35 \mathrm{~cm} \times 10.0 \mathrm{~cm}$ & 4.48 & 63.03 & 34.53 & 7.56 & 1318.33 \\
\hline
\end{tabular}

American Research Journal of Agriculture

Page 2 
Yield and Quality of White Jute (var. BJRI Deshi Pat 8) Seed as Influenced by LinexPlant Spacing in Different Growing Areas of Bangladesh

\begin{tabular}{|c|c|c|c|c|c|c|}
\hline \hline $\mathrm{S}_{5}$ & $35 \mathrm{~cm} \times 12.5 \mathrm{~cm}$ & 5.95 & 75.16 & 37.34 & 9.49 & 1398.33 \\
\hline $\mathrm{S}_{6}$ & $35 \mathrm{~cm} \times 15.0 \mathrm{~cm}$ & 4.21 & 42.70 & 31.01 & 6.22 & 1221.00 \\
\hline $\mathrm{S}_{7}$ & $40 \mathrm{~cm} \times 10.0 \mathrm{~cm}$ & 5.90 & 73.90 & 36.93 & 9.22 & 1379.33 \\
\hline $\mathrm{S}_{8}$ & $40 \mathrm{~cm} \times 12.5 \mathrm{~cm}$ & 4.08 & 52.43 & 33.73 & 6.62 & 1285.00 \\
\hline $\mathrm{S}_{9}$ & $40 \mathrm{~cm} \times 15.0 \mathrm{~cm}$ & 4.14 & 41.10 & 29.34 & 5.82 & 1187.67 \\
\hline $\mathrm{S}_{10}$ & $45 \mathrm{~cm} \times 10.0 \mathrm{~cm}$ & 4.94 & 67.43 & 34.88 & 8.36 & 1336.33 \\
\hline $\mathrm{S}_{11}$ & $45 \mathrm{~cm} \times 12.5 \mathrm{~cm}$ & 4.74 & 47.43 & 33.37 & 6.42 & 1269.33 \\
\hline $\mathrm{S}_{12}$ & $45 \mathrm{~cm} \times 15.0 \mathrm{~cm}$ & 3.81 & 36.63 & 21.38 & 4.16 & 1074.33 \\
\hline \multicolumn{2}{|l|}{ LSD $_{(0.05)}$} & $\mathrm{NS}$ & 3.635 & 0.577 & 0.631 & 37.81 \\
\hline
\end{tabular}

Legend: $N S=$ Not-significant, $P H=$ Plant height, $S W=$ Seed weight, $S Y=$ Seed yield

Table2. Effect of spacing on seed quality contributing characters of white jute at Manikganj

\begin{tabular}{|c|c|c|c|c|}
\hline \multicolumn{2}{|c|}{ Treatment (Spacing) } & \multirow{2}{*}{1000 SW (g) } & \multirow{2}{*}{ Ger (\%) } & \multirow{2}{*}{$\operatorname{Mos}(\%)$} \\
\hline & cm & & & \\
\hline $\mathrm{S}_{1}$ & $30 \mathrm{~cm} \times 10.0 \mathrm{~cm}$ & 2.73 & 75.33 & 9.55 \\
\hline $\mathrm{S}_{2}$ & $30 \mathrm{~cm} \times 12.5 \mathrm{~cm}$ & 2.85 & 82.67 & 10.24 \\
\hline $\mathrm{S}_{3}$ & $30 \mathrm{~cm} \times 15.0 \mathrm{~cm}$ & 3.17 & 91.33 & 10.46 \\
\hline $\mathrm{S}_{4}$ & $35 \mathrm{~cm} \times 10.0 \mathrm{~cm}$ & 3.01 & 85.67 & 10.30 \\
\hline $\mathrm{S}_{5}$ & $35 \mathrm{~cm} \times 12.5 \mathrm{~cm}$ & 3.34 & 92.33 & 10.72 \\
\hline $\mathrm{S}_{6}$ & $35 \mathrm{~cm} \times 15.0 \mathrm{~cm}$ & 2.77 & 78.00 & 9.84 \\
\hline $\mathrm{S}_{7}$ & $40 \mathrm{~cm} \times 10.0 \mathrm{~cm}$ & 3.23 & 91.67 & 10.57 \\
\hline $\mathrm{S}_{8}$ & $40 \mathrm{~cm} \times 12.5 \mathrm{~cm}$ & 2.84 & 79.67 & 10.12 \\
\hline $\mathrm{S}_{9}$ & $40 \mathrm{~cm} \times 15.0 \mathrm{~cm}$ & 2.76 & 76.67 & 9.64 \\
\hline$S_{10}$ & $45 \mathrm{~cm} \times 10.0 \mathrm{~cm}$ & 3.13 & 89.00 & 10.36 \\
\hline$S_{11}$ & $45 \mathrm{~cm} \times 12.5 \mathrm{~cm}$ & 2.79 & 79.67 & 10.02 \\
\hline $\mathrm{S}_{12}$ & $45 \mathrm{~cm} \times 15.0 \mathrm{~cm}$ & 2.68 & 74.33 & 9.04 \\
\hline \multicolumn{2}{|c|}{$\mathrm{LSD}_{(0.05)}$} & NS & 2.732 & NS \\
\hline
\end{tabular}

Legend: NS=Not-significant, Ger=Germination, Mos=Moisture

Table3. Effect of spacing on seed yield and yield contributing characters of white jute at Rangpur

\begin{tabular}{|c|c|c|c|c|c|c|}
\hline \multicolumn{2}{|c|}{ Treatment (Spacing) } & \multirow{2}{*}{$\begin{array}{l}\text { Branch } \\
\text { plant }^{-1}\end{array}$} & \multirow{2}{*}{$\begin{array}{l}\text { Pods } \\
\text { plant }^{-1}\end{array}$} & \multirow{2}{*}{$\begin{array}{l}\text { Seed } \\
\text { pod }^{-1}\end{array}$} & \multirow{2}{*}{$\begin{array}{c}\text { SW } \\
\text { plant }^{-1}(g)\end{array}$} & \multirow{2}{*}{ SY $\left(\mathrm{kgha}^{-1}\right)$} \\
\hline & $\mathbf{c m}$ & & & & & \\
\hline $\mathrm{S}_{1}$ & $30 \mathrm{~cm} \times 10.0 \mathrm{~cm}$ & 3.84 & 32.91 & 31.99 & 5.48 & 642.20 \\
\hline $\mathrm{S}_{2}$ & $30 \mathrm{~cm} \times 12.5 \mathrm{~cm}$ & 5.04 & 42.64 & 40.72 & 6.74 & 952.73 \\
\hline $\mathrm{S}_{3}$ & $30 \mathrm{~cm} \times 15.0 \mathrm{~cm}$ & 5.89 & 53.24 & 46.06 & 9.01 & 1107.30 \\
\hline $\mathrm{S}_{4}$ & $35 \mathrm{~cm} \times 10.0 \mathrm{~cm}$ & 5.64 & 48.84 & 42.56 & 7.68 & 1007.63 \\
\hline $\mathrm{S}_{5}$ & $35 \mathrm{~cm} \times 12.5 \mathrm{~cm}$ & 5.94 & 59.44 & 46.89 & 9.61 & 1164.73 \\
\hline $\mathrm{S}_{6}$ & $35 \mathrm{~cm} \times 15.0 \mathrm{~cm}$ & 4.51 & 37.71 & 38.39 & 6.34 & 767.93 \\
\hline $\mathrm{S}_{7}$ & $40 \mathrm{~cm} \times 10.0 \mathrm{~cm}$ & 5.84 & 57.24 & 46.72 & 9.34 & 1128.17 \\
\hline $\mathrm{S}_{8}$ & $40 \mathrm{~cm} \times 12.5 \mathrm{~cm}$ & 5.38 & 48.44 & 41.56 & 7.28 & 1002.03 \\
\hline
\end{tabular}


Yield and Quality of White Jute (var. BJRI Deshi Pat 8) Seed as Influenced by LinexPlant Spacing in Different Growing Areas of Bangladesh

\begin{tabular}{|c|c|c|c|c|c|c|}
\hline \hline $\mathrm{S}_{9}$ & $40 \mathrm{~cm} \times 15.0 \mathrm{~cm}$ & 4.18 & 36.78 & 36.06 & 5.94 & 717.60 \\
\hline $\mathrm{S}_{10}$ & $45 \mathrm{~cm} \times 10.0 \mathrm{~cm}$ & 5.7 & 50.71 & 45.39 & 8.48 & 1057.37 \\
\hline $\mathrm{S}_{11}$ & $45 \mathrm{~cm} \times 12.5 \mathrm{~cm}$ & 4.78 & 40.18 & 40.56 & 6.54 & 946.73 \\
\hline $\mathrm{S}_{12}$ & $45 \mathrm{~cm} \times 15.0 \mathrm{~cm}$ & 2.91 & 28.98 & 30.06 & 4.28 & 612.13 \\
\hline \multicolumn{2}{|c|}{$\mathrm{LSD}_{(0.05)}$} & NS & 2.878 & 1.983 & 0.631 & 53.06 \\
\hline
\end{tabular}

Legend: $N S=$ Not-significant, $P H=$ Plant height, $S W=$ Seed weight, $S Y=$ Seed yield

At Rangpur area the yield and yield contributing characters of the study material were differed significantly except branch plant ${ }^{-1}$ due to different plant spacing (Table 3). The highest yield $1164.73 \mathrm{kgha}^{-1}$ was obtained from the spacing $35 \mathrm{~cm} \times 12.5 \mathrm{~cm}$ which was followed by the spacing $40 \mathrm{~cm} \times 10.0 \mathrm{~cm}\left(1128.17 \mathrm{kgha}^{-1}\right)$ and $30 \mathrm{~cm} \times 15 \mathrm{~cm}$ (1107.30 $\mathrm{kgha}^{-1}$ ). Yield contributing characters were gave similar results according to the seed yield at Rangpur (Table 3). At Rangpur germination percent differed significantly due to spacing, however, $1000 \mathrm{SW}$ (g) and moisture percentage were insignificant. The highest germination was observed $92.67 \%$ from $35 \mathrm{~cm} \times 12.5 \mathrm{~cm}$ spacing which was followed by the spacing $40 \mathrm{~cm} \times 10.0 \mathrm{~cm}(91.67 \%)$ and $30 \mathrm{~cm} \times 15 \mathrm{~cm}(89.33 \%)$ (Table 4). These results were in agreement with Islam (2009), Islam (2010) and Islam and Rahman (2008).

Table4. Effect of spacing on seed quality characters of white jute at Rangpur

\begin{tabular}{|c|c|c|c|c|}
\hline \multicolumn{2}{|c|}{ Treatment (Spacing) } & \multirow{2}{*}{$\mathbf{1 0 0 0}$ SW (g) } & \multirow{2}{*}{ Ger (\%) } & \multirow{2}{*}{ Mos (\%) } \\
\hline$y^{2} \mathrm{~S}_{1}$ & $30 \mathrm{~cm} \times 10.0 \mathrm{~cm}$ & 2.74 & 78.33 & 10.22 \\
\hline $\mathrm{S}_{2}$ & $30 \mathrm{~cm} \times 12.5 \mathrm{~cm}$ & 3.01 & 82.33 & 10.48 \\
\hline $\mathrm{S}_{3}$ & $30 \mathrm{~cm} \times 15.0 \mathrm{~cm}$ & 3.28 & 89.33 & 10.90 \\
\hline $\mathrm{S}_{4}$ & $35 \mathrm{~cm} \times 10.0 \mathrm{~cm}$ & 3.15 & 85.67 & 10.67 \\
\hline $\mathrm{S}_{5}$ & $35 \mathrm{~cm} \times 12.5 \mathrm{~cm}$ & 3.43 & 92.67 & 10.77 \\
\hline $\mathrm{S}_{6}$ & $35 \mathrm{~cm} \times 15.0 \mathrm{~cm}$ & 2.97 & 79.67 & 10.28 \\
\hline $\mathrm{S}_{7}$ & $40 \mathrm{~cm} \times 10.0 \mathrm{~cm}$ & 3.36 & 91.67 & 10.82 \\
\hline $\mathrm{S}_{8}$ & $40 \mathrm{~cm} \times 12.5 \mathrm{~cm}$ & 3.05 & 83.67 & 10.34 \\
\hline $\mathrm{S}_{9}$ & $40 \mathrm{~cm} \times 15.0 \mathrm{~cm}$ & 2.90 & 78.67 & 10.06 \\
\hline $\mathrm{S}_{10}$ & $45 \mathrm{~cm} \times 10.0 \mathrm{~cm}$ & 3.25 & 87.00 & 10.63 \\
\hline $\mathrm{S}_{11}$ & $45 \mathrm{~cm} \times 12.5 \mathrm{~cm}$ & 3.01 & 80.33 & 9.98 \\
\hline $\mathrm{S}_{12}$ & $45 \mathrm{~cm} \times 15.0 \mathrm{~cm}$ & 2.70 & 77.33 & 9.55 \\
\hline \multicolumn{2}{|l|}{$\mathrm{LSD}_{(0.05)}$} & $\mathrm{NS}$ & 3.385 & $\mathrm{NS}$ \\
\hline
\end{tabular}

Legend: $N S$ = Not-significant, Ger = Germination, Mos = Moisture

Table5. Effect of spacing on seed yield and yield contributing characters of white jute at Jessore

\begin{tabular}{|c|c|c|c|c|c|c|}
\hline \multicolumn{2}{|c|}{ Treatment (Spacing) } & \multirow{2}{*}{$\begin{array}{l}\text { Branch } \\
\text { plant }^{-1}\end{array}$} & \multirow{2}{*}{$\begin{array}{l}\text { Pods } \\
\text { plant }^{-1}\end{array}$} & \multirow{2}{*}{$\begin{array}{l}\text { Seed } \\
\operatorname{pod}^{-1}\end{array}$} & \multirow{2}{*}{$\begin{array}{c}\text { SW } \\
\text { plant }^{-1}(\mathrm{~g})\end{array}$} & \multirow{2}{*}{$\begin{array}{c}\text { SY } \\
\left(\text { kgha- }^{-1}\right)\end{array}$} \\
\hline & $\mathbf{c m}$ & & & & & \\
\hline $\mathrm{S}_{1}$ & $30 \mathrm{~cm} \times 10.0 \mathrm{~cm}$ & 2.78 & 26.10 & 31.69 & 4.38 & 673.67 \\
\hline $\mathrm{S}_{2}$ & $30 \mathrm{~cm} \times 12.5 \mathrm{~cm}$ & 3.18 & 31.17 & 35.67 & 5.51 & 927.00 \\
\hline $\mathrm{S}_{3}$ & $30 \mathrm{~cm} \times 15.0 \mathrm{~cm}$ & 3.55 & 36.64 & 37.62 & 6.44 & 1053.67 \\
\hline $\mathrm{S}_{4}$ & $35 \mathrm{~cm} \times 10.0 \mathrm{~cm}$ & 3.28 & 32.17 & 35.87 & 5.88 & 945.33 \\
\hline $\mathrm{S}_{5}$ & $35 \mathrm{~cm} \times 12.5 \mathrm{~cm}$ & 4.05 & 39.70 & 39.00 & 7.24 & 1197.00 \\
\hline $\mathrm{S}_{6}$ & $35 \mathrm{~cm} \times 15.0 \mathrm{~cm}$ & 3.11 & 30.17 & 35.25 & 5.41 & 884.33 \\
\hline $\mathrm{S}_{7}$ & $40 \mathrm{~cm} \times 10.0 \mathrm{~cm}$ & 3.65 & 37.80 & 37.99 & 6.88 & 1097.00 \\
\hline
\end{tabular}


Yield and Quality of White Jute (var. BJRI Deshi Pat 8) Seed as Influenced by LinexPlant Spacing in Different Growing Areas of Bangladesh

\begin{tabular}{|c|c|c|c|c|c|c|}
\hline \hline $\mathrm{S}_{8}$ & $40 \mathrm{~cm} \times 12.5 \mathrm{~cm}$ & 3.05 & 29.00 & 34.73 & 5.11 & 860.33 \\
\hline $\mathrm{S}_{9}$ & $40 \mathrm{~cm} \times 15.0 \mathrm{~cm}$ & 2.95 & 28.17 & 33.35 & 4.88 & 790.33 \\
\hline $\mathrm{S}_{10}$ & $45 \mathrm{~cm} \times 10.0 \mathrm{~cm}$ & 3.35 & 36.00 & 36.27 & 6.19 & 1000.33 \\
\hline $\mathrm{S}_{11}$ & $45 \mathrm{~cm} \times 12.5 \mathrm{~cm}$ & 2.88 & 27.10 & 32.27 & 4.78 & 723.67 \\
\hline $\mathrm{S}_{12}$ & $45 \mathrm{~cm} \times 15.0 \mathrm{~cm}$ & 2.71 & 24.84 & 28.89 & 3.68 & 592.00 \\
\hline \multicolumn{2}{|c|}{ LSD $_{(0.05)}$} & $\mathrm{NS}$ & 0.310 & 2.173 & 0.131 & 85.31 \\
\hline
\end{tabular}

Legend: $N S=$ Not-significant, $P H=$ Plant height, $S W=$ Seed weight, $S Y=$ Seed yield

At Jessore results showed that all the yield and yield contributing characters were differed significantly except Branch plant ${ }^{-1}$ due to different plant spacing (Table 5). The highest seed yield of $1197.00 \mathrm{kgha}^{-1}$ was obtained in Jessore from the spacing $35 \mathrm{~cm} \times 12.5 \mathrm{~cm}$ which was followed by the spacing $40 \mathrm{~cm} \times 10 \mathrm{~cm}\left(1097 \mathrm{kgha}^{-1}\right)$ and $30 \mathrm{~cm} \times 15 \mathrm{~cm}\left(1053.67 \mathrm{kgha}^{-1}\right)$. All other yield contributing characters were gave similar results according to the seed yield (Table 5). At Jessore germination percent differed significantly due to spacing, however, 1000 SW (g) and moisture percentage were insignificant (Table 6). The highest germination was observed $91.33 \%$ at Jessore from $35 \mathrm{~cm} \times 12.5 \mathrm{~cm}$ specing which was followed by the spacing $40 \mathrm{~cm} \times 10.0 \mathrm{~cm}(89.33 .67 \%)$ and $30 \mathrm{~cm} \times 15 \mathrm{~cm}(86.33 \%)$ (Table 6). Results were in agreement with Islam (2009), Islam (2010) and Islam and Rahman (2008).

Table6. Effect of spacing on seed quality characters of white jute at Jessore

\begin{tabular}{|c|c|c|c|c|}
\hline \multicolumn{2}{|c|}{ Treatment (Spacing) } & \multirow{2}{*}{$\mathbf{1 0 0 0} \mathbf{~ S W ~ ( g ) ~}$} & Ger (\%) & \multirow{2}{*}{ Mos (\%) } \\
\hline $\mathrm{S}_{1}$ & $30 \mathrm{~cm} \times 10.0 \mathrm{~cm}$ & 2.86 & 77.00 & 9.47 \\
\hline $\mathrm{S}_{2}$ & $30 \mathrm{~cm} \times 12.5 \mathrm{~cm}$ & 3.13 & 81.33 & 10.38 \\
\hline $\mathrm{S}_{3}$ & $30 \mathrm{~cm} \times 15.0 \mathrm{~cm}$ & 3.20 & 86.33 & 10.71 \\
\hline $\mathrm{S}_{4}$ & $35 \mathrm{~cm} \times 10.0 \mathrm{~cm}$ & 3.17 & 82.67 & 10.43 \\
\hline $\mathrm{S}_{5}$ & $35 \mathrm{~cm} \times 12.5 \mathrm{~cm}$ & 3.31 & 91.33 & 11.06 \\
\hline $\mathrm{S}_{6}$ & $35 \mathrm{~cm} \times 15.0 \mathrm{~cm}$ & 3.06 & 80.33 & 10.33 \\
\hline $\mathrm{S}_{7}$ & $40 \mathrm{~cm} \times 10.0 \mathrm{~cm}$ & 3.27 & 89.33 & 10.77 \\
\hline $\mathrm{S}_{8}$ & $40 \mathrm{~cm} \times 12.5 \mathrm{~cm}$ & 2.98 & 79.33 & 10.19 \\
\hline $\mathrm{S}_{9}$ & $40 \mathrm{~cm} \times 15.0 \mathrm{~cm}$ & 2.92 & 78.33 & 9.80 \\
\hline $\mathrm{S}_{10}$ & $45 \mathrm{~cm} \times 10.0 \mathrm{~cm}$ & 3.19 & 83.67 & 10.48 \\
\hline $\mathrm{S}_{11}$ & $45 \mathrm{~cm} \times 12.5 \mathrm{~cm}$ & 2.89 & 78.00 & 9.65 \\
\hline $\mathrm{S}_{12}$ & $45 \mathrm{~cm} \times 15.0 \mathrm{~cm}$ & 2.74 & 75.67 & 9.25 \\
\hline \multicolumn{2}{|c|}{$\mathrm{LSD}_{(0.05)}$} & $\mathrm{NS}$ & 1.486 & $\mathrm{NS}$ \\
\hline
\end{tabular}

Legend: $N S=$ Not-significant, Ger = Germination, Mos = Moisture

\section{CONCLUSION}

From the above results it could be concluded that the highest seed yield and germination were recorded at Manikganj, Rangpur and at Jessore from the spacing treatment $35 \mathrm{~cm} \times 12.5 \mathrm{~cm}$, was followed by $40 \mathrm{~cm} \times 10 \mathrm{~cm}$ and $30 \mathrm{~cm} \times 15 \mathrm{~cm}$. The newly released white jute var. BJRI Deshi Pat 8 could be produced for higher seed yield with line $\times$ plant spacing of $35 \mathrm{~cm} \times 12.5 \mathrm{~cm}$. 
Yield and Quality of White Jute (var. BJRI Deshi Pat 8) Seed as Influenced by LinexPlant Spacing in Different Growing Areas of Bangladesh

Conflict of interest: The authors declare no conflict of interest.

Author contributions: Islam, M.M. designed, performed research and wrote the manuscript; Ali, M.S., Hossain, M.S. \& Rahman, M.S analyzed data.

\section{REFERENCES}

Alom, M.F.; Alam, A.T.M.M.; Ghosh, R.K.; Zia, M.I.K. and Islam, M. (2010) Planting time and sowing effects on jute seed yield. Seed Tech. J. 1: 55-62.

Chanda, S.C.; Kader, M.A., Islam, M. M. and Haque, M.M. 1999. Effects of Sowing Dates on the Yield and Quality of Jute and Kenaf Seeds. Bangladesh J. Seed Scie. \& Technology. 3(1\&2): 11-16.

Gomez, A. K. and Gomez, A. A. 1984. Statistical Procedure for Agric. Res. Second Edn. Intnl.Rice Res. Inst. Manila. Philippines. pp. 139-207

IJSG. 2012. World Jute \& Kenaf Statistics: at a Glance 2012, Jute, Kenaf Other Bast and Hard Fibres: Farm to Fashion, Published by: International Jute Study Group (IJSG) IJSG Secretariat, 145, Monipuripara, Near Farmgate, Tejgoan, Dhaka-1215, Bangladesh, E-mail: info@jute.org, Web: www.jute.org.

Islam, M.M. 2009. Jute seed technology. $1^{\text {st }}$ edition. September 2009. Pub. by Md. Mahmudul Islam, 397, Middle Monipur, Mirpur, Dhaka-1216. College Gate Book Binding and Printing, Mohamadpur, Dhaka. pp.160.

Islam, M.M. 2010. Technological advances in off-season jute seed production. J. Expt. Biosci. 1(1): 75-82.

Islam, M.M. and Rahman, M. 2008. In: Hand book on agricultural Technologies of Jute, Kenaf and Mesta crops. Bangladesh Jute Research Institute, Manikmia Avenue, Dhaka-1207, Bangladesh. p.92.

Khan, A.; Samad,M.A.; Hossain,M.A. Islam, M.M. and Rabbany, G. 1997. Effect of sowing date and variety on olitorius jute seed production. Bangladesh J. Jute and Fib. Res. 22(1\&2): 19-25.

Rahman, M. M.; Rahman, M. L.; Azad, A. K.; Rahman, M.; and Hussain, M. M. 2003. Impact of component technologies in jute production. Bangladesh J. Jute Fib. Res. 24(1-8): 21-26.

Talukder, F.A.H.; Islam, M.M.; Chanda, S.C.; Ahmed, I. and Ahmed, Z. 2001. Phenology of Jute, Kenaf and Roselle Seed Crops at Different Dates of Sowing. Pakistan J. Biol. Sci. 4 (11): 1316-1318.

Citation: Md. Mahbubul Islam, Md. Shahadat Hossain et al. "Yield And Quality Of White Jute (Var. Bjri Deshi Pat 8) Seed As Influenced By LinexPlant Spacing In Different Growing Areas Of Bangladesh." American Research Journal Of Agriculture, vol 3, no. 1, 2017, pp. 1-6.

Copyright (c) 2017 Md. Mahbubul Islam, Md. Shahadat Hossain et al. This is an open access article distributed under the Creative Commons Attribution License, which permits unrestricted use, distribution, and reproduction in any medium, provided the original work is properly cited. 